\title{
Time-Varying Effect of Gold and Crude Oil prices to Stock Price Index
}

\author{
Sukrit Thongkairat ${ }^{1}$ and Roengchai Tansuchat ${ }^{2, *}$ \\ ${ }^{1}$ Faculty of Economics, Chiang Mai University, Chiang Mai, Thailand. \\ ${ }^{2}$ Faculty of Economics, Chiang Mai University, Chiang Mai, Thailand. \\ *Email: roengchaitan@gmail.com
}

\begin{abstract}
This paper proposes time-varying effect of gold and crude oil price to stock price index with Markov switching state space model which provided the time-varying coefficients to analyze the time-varying behaviors of the stock index. A Bayesian approach is employed to predict the parameters of the model whereas Kalman filter is applied to predict the time-varying coefficients in each regime. The stock price index, gold price and oil price dataset from 1st April 1996 to 31st March 2016 with the total 5,219 observations were used. The result shows that the proposed model is able to significantly capture the real economic situation and it can capture the movement of the coefficients in each period.
\end{abstract}

Keywords: Time-Varying coefficient, Markov Switching, Kalman filter, Bayesian estimation

\section{Introduction}

Stock markets are the most important asset in the economy because it is basically considered as one of the crucial determinants of economic outputs. In the empirical research studies, there are many evidences presenting the relationships or dependences between stock price index and other commodity market particularly gold market and crude oil market. For example, Akgül et al [1], Basher et al [2] and Philip et al [3] have confirmed the evidence of the relationship between oil price, gold and stock price index. Fig. 1 shows the co-movements among gold and crude oil price that seem to be inversely related to the S\&P 500 index.

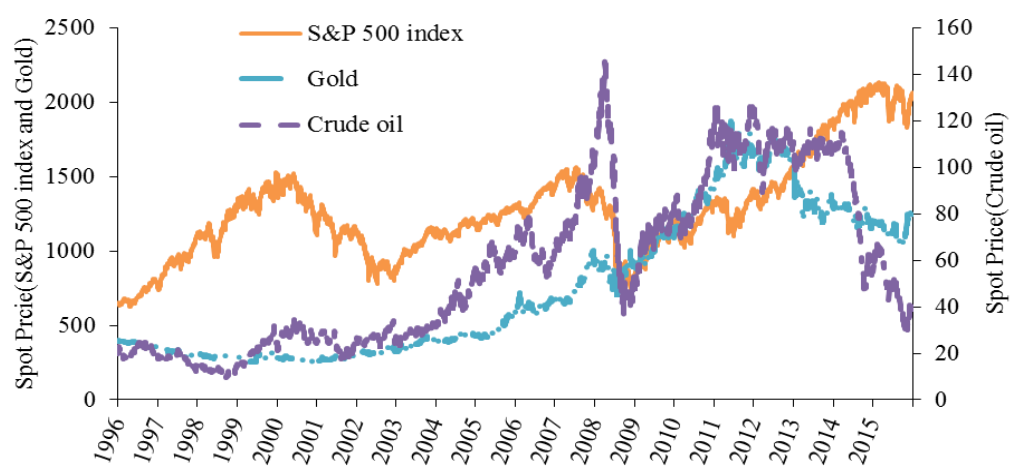

Figure 1 S\&P 500 Index, WTI spot FOB US\$/BBL (Crude oil), and Gold US\$/OZ, 1996 - 2016 The co-movements among these price indexes have attracted to increase the attention of financial managers and researchers to examine their linkages because the relationships are importantly implicated in economy as well as in financial markets. In addition, Akgül et al [1], Basher et al [2], Philip et al [3] indicated that the relationships of gold and crude oil price to stock price index are differently in the different situation or regimes.

The research study is to consider the time-varying effect of gold and crude oil price to stock price index by applying Markov switching state-space model. This model was introduced by Kim and Nelson [4] and Kim [5] uses to deal with the problems of parameters based on the ordinary Markov switching which is constantly over time, that in turn making the estimated parameters are wrong. This concept has spread to other research studies in the works of Filardo[6], Paravee [7] and Kang [8]. Therefore, the paper is structured as follows. In section 2 provides a description of the methodology and dataset. Section 3 presents the empirical results and finally the last section is the conclusions. 


\section{Methodology and data}

\subsection{Methodology}

\subsubsection{Markov Switching Regression with Time-Varying Coefficients}

In 1997, West and Harrison [9] proposed the time-varying model that has been frequently used to capture heterogeneous dynamics of the data varying over time. Accordingly, in this paper, time-varying model will be integrated to a Markov switching model of Hamilton [10] and purposed a Markov switching regression with time-varying coefficients in each regime. Two state regimes are postulated, namely downturn market and upturn market to basically cover switching states of the economy. Markov switching can be written in the following form:

$$
y_{t}=a_{\left(\mathrm{S}_{t}\right)}+b_{t,\left(\mathrm{~S}_{t}\right)} X_{t}^{\prime}+e_{t}
$$

where $y_{t}$ is a vector of dependent variable, $X_{t}$ is a matrix of independent variable, $a_{\left(\mathrm{S}_{t}\right)}$ and $b_{t,\left(\mathrm{~S}_{t}\right)}$ are regime dependent parameters, respectively. The $e_{t}$ is observational errors which are allowed to vary over time according to these following equations.

$$
\begin{aligned}
& \left(a_{\left(S_{t}\right), t+1}-\bar{a}_{\left(S_{t}\right)}\right)=P_{t,\left(S_{t}\right)}\left(a_{t,\left(S_{t}\right)}-\bar{a}_{t,\left(S_{t}\right)}\right)+u_{t+1} \\
& \left(b_{\left(S_{t}\right), t+1}-\bar{b}_{\left(S_{t}\right)}\right)=F_{t,\left(S_{t}\right)}\left(b_{t,\left(S_{t}\right)}-\bar{b}_{t,\left(S_{t}\right)}\right)+v_{t+1}
\end{aligned}
$$

The eq. (2) is the time-varying equations, in which the term $\bar{a}_{t,\left(S_{t}\right)}$ and $\bar{b}_{t,\left(S_{t}\right)}$ are the average of the steady state coefficient vector and intercept term, respectively, which are regime dependent. Following Hamilton [11] let $\tilde{b}_{t,\left(S_{t}\right)}=\left(b_{t,\left(S_{t}\right)}-\bar{b}_{t,\left(S_{t}\right)}\right)$ and $\tilde{a}_{t,\left(S_{t}\right)}=\left(a_{t,\left(S_{t}\right)}-\bar{a}_{t,\left(S_{t}\right)}\right)$ thus, we can rewrite eq. 1 as

$$
y_{t}=\bar{a}_{\left(S_{t}\right)}+a_{\left(S_{t}\right)}+\bar{b}_{t,\left(S_{t}\right)} X_{t,\left(S_{t}\right)}^{\prime}+b_{t,\left(S_{t}\right)} X_{t,\left(S_{t}\right)}^{\prime}+e_{t}
$$

where the term $S_{t}$ represents a state variable which is governed by the first order Markov chain. Then, the transition probability $(Q)$ can be defined by $p_{i j}=\operatorname{Pr}\left(S_{t+1}=j \mid S_{t}=i\right)$ and $\sum_{j=1}^{k} p_{i j}=1$, $i, j=1, \ldots, \mathrm{k}$, where $p_{i j}$ is the probability of regime $i$ followed by regime $j$, and it is convenient to collect all transition probabilities in the transition matrix $Q$,

$$
Q=\left[\begin{array}{cccc}
p_{11} & p_{21} & \cdots & p_{k 1} \\
p_{12} & p_{22} & \cdots & p_{k 2} \\
\vdots & \vdots & \ddots & \vdots \\
p_{1 k} & p_{2 k} & \cdots & p_{k k}
\end{array}\right]
$$

\subsubsection{The Kalman Filter and Estimation of Parameter}

Kalman filter is a recursive procedure used to estimate unobserved parameters based on $\theta_{\left(S_{t}\right)}=\left(a_{\left(S_{t}\right)}, b_{\left(S_{t}\right)}\right)$ available information in the dataset $(\psi)$. Briefly, the Kalman filter consists of 2 step estimations:

1. Prediction: In this step, the prediction of $y_{t}$ is based on $y_{t-1}$, where $y_{t-1}$ is computed from the expectation of $E\left[a_{\left(S_{t}\right)} \mid \psi_{t-1}\right]$ and $E\left[b_{\left(S_{t}\right)} \mid \psi_{t-1}\right]$

2. Updating: Updating the inference about the state vector incorporating Kalman gain matrix and the prediction error. Once $y_{t}$ is realized at the end of time $t$, the prediction error can be computed by $v_{t-1}=y_{t}-y_{t \mid t-1}$. Then $b_{\left(\mathrm{S}_{t}\right)}$ based on $\psi_{t}$ can be estimated by the following form:

$$
b_{\left(\mathrm{S}_{t}\right)_{t \mid}}=b_{\left(\mathrm{S}_{t}\right)_{t \mid-1}}+W_{t\left(\mathrm{~S}_{t}\right)_{t \mid t-1}}
$$

where $W_{\left(\mathrm{S}_{t}\right)}$ is a weight assigned to new information about $a_{\left(S_{t}\right)}$ and $b_{\left(S_{t}\right)}$ contained in the predicted error. (Kim [4]) 


\subsubsection{Prior Distribution and the Posterior}

In this study, we use the Normal distribution for the prior probability $G \sim N\left(\overline{\mathrm{G}}, \sigma_{\bar{G}}\right)$ and Inverse gamma prior for sigma $\sigma_{v}^{2} \sim I W(c / 2, d / 2)$, where $c / 2$ and $d / 2$ are the shape parameter and scale parameter, respectively. The weak Dirichlet priors with preference for staying in its own regime is chosen for the transition probabilities $\tilde{Q} \sim \operatorname{Dirichlet}\left(q_{1}, q_{2}, q_{3}\right)$ where $q_{i}$ is the scale parameter. Then, the Gibbs sampling is used to run the sampler for 10,000 iterations, with a burn-in of 2,000 iterations. Finally, 8,000 estimated parameter sets are summed, and then to be divided by 8,000 in order to obtain the estimated mean parameters of the dataset and gotten $\widehat{G}$ and $\widehat{\mathcal{S}}$. The posterior estimation can be computed by combining together these priors with the likelihood function using Bayes theorem as follows:

Posterior probability $\propto$ likelihood $\times$ Prior probability.

The Markov switching linear regression with time-varying can be written in the following form:

$$
y_{t}=\bar{a}_{\left(S_{t}\right)}+\bar{b}_{t,\left(S_{t}\right)} X_{t,\left(S_{t}\right)}^{\prime}+\Theta_{t,\left(S_{t}\right)} X_{t,\left(S_{t}\right)}^{\prime}+e_{t}, \Theta_{t}=G \Theta_{t-1}+\eta_{t}
$$

where $P(\varsigma, G)$ is denoted the prior of $G$ and $\varsigma$; and $P\left(y_{t}, X_{t} \mid \varsigma, \Theta_{\left(S_{t}\right)}, G\right)$. A Likelihood function can be formed transformed to logarithm function as follows:

$$
\ln L=-\frac{1}{2} \sum_{t=1}^{T} \ln \left(2 \pi\left|f_{t \mid t-1}\right|\right)-\frac{1}{2} \eta_{t \mid t-1}^{\prime} f_{t \mid t-1}^{-1} \eta_{t \mid t-1}
$$

Following Kim and Nelson [4], the Gibbs sampling algorithm is conducted to draw the parameter set, $G$ and $\Theta$ to estimate each parameter, the block optimization is employed.

\subsection{Data}

The daily dataset used in the study consists of the stock price index from S\&P 500 index price $\left(P^{\text {stock }}\right)$, crude oil prices ( $\left.P^{\text {oil }}\right)$ and gold prices $\left(P^{\text {gold }}\right)$ in which was collected from 1st April 1996 to 31 st March 2016 covering the total observations of 5,219. Prior to estimation, we check the stationary of the dataset by testing the Bayes factor as proposed in Wang and Ghosh [12]. Results of Bayes factor testing were showed in table 1 . The value of the variables of the Bayes factor is less than 1. Thus, all variables can reject the hypothesis of $\left(P\left(\theta=1 \mid y_{t}\right)\right)$ and accept $P\left(0<\theta<1 \mid y_{t}\right)$, meaning that they are stationary and can be continually used to modeling.

Table 1 Unit Root Test

\begin{tabular}{ccc}
\hline Variable & Bayes Factor & Result \\
\hline$P^{\text {stock }}$ & 0.9998112 & Stationary \\
$P^{\text {gold }}$ & 0.9997499 & Stationary \\
$P^{\text {oil }}$ & 0.9992574 & Stationary \\
\hline
\end{tabular}

\section{Empirical results}

The empirical results demonstrate that the time-varying coefficients describing the behavior of the stock prices index can appropriately capture the movement of the coefficients over time. The results of Filtered probabilities are shown in Fig. 2. The probability values vary between 0 and 1 , and accordingly the observation is classified in the Regime 1 if $\operatorname{Pr}\left(S_{t}=1 \mid y_{t}\right)>0.5$ and in the Regime 2 if $\operatorname{Pr}\left(S_{t}=1 \mid y_{t}\right) \leq 0.5$ [10].

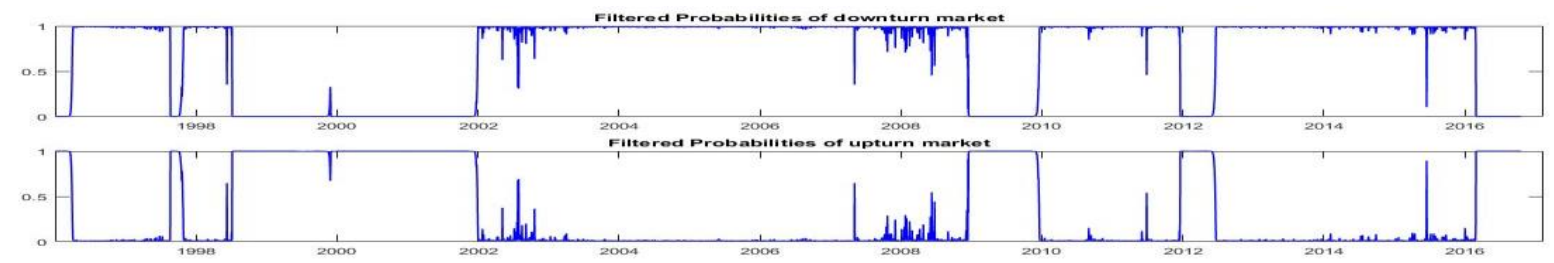

Figure 2 Filtered probabilities of downturn market (Top) and upturn market (Bottom) 
Tables 2 shows time-varying parameters with Markov switching Regression. The parameters of the variables are statistically significant. The coefficient of the gold price is positive and implied that the higher the gold price, increasing $1 \%$, the higher the stock price index rising $0.006923 \%$. Similarly, if the crude oil price drops $1 \%$, it will cause to diminish in the stock price index $0.027198 \%$. As the result, the estimation can be observed completely and graphically in Figure 3 where all coefficients of the variables are varying over time.

Tables 2 Time-Varying Parameter with Markov switching Estimates

\begin{tabular}{cccc}
\hline Variable & coefficients & Std. deviation & t-statistic \\
\hline Intercept & 9.445824 & 0.037448 & 252.240821 \\
$P^{\text {gold }}$ & 0.006923 & 0.037448 & 21.611999 \\
$P^{\text {oil }}$ & 0.027198 & 0.003000 & 9.066800 \\
\hline
\end{tabular}
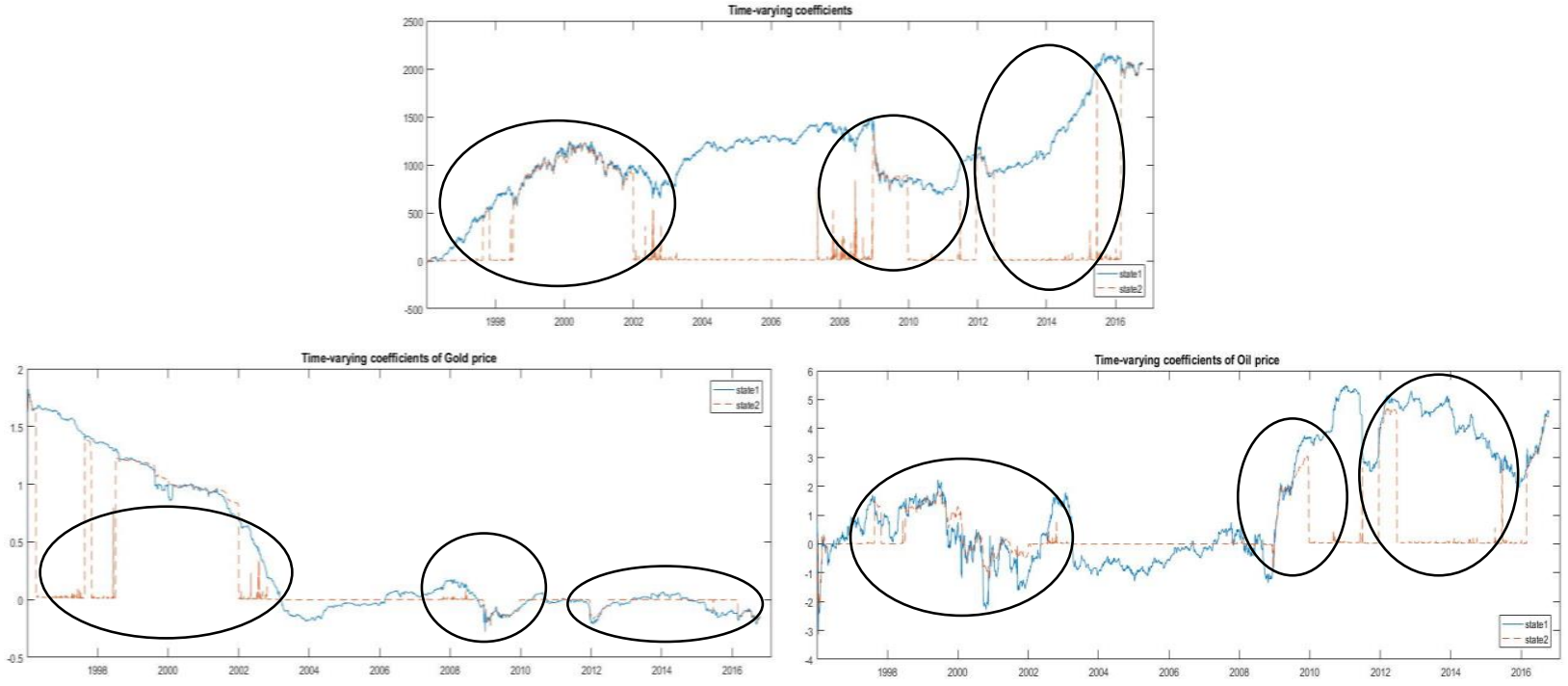

Figure 3 Time-varying coefficients of Stock prices index (Top), Gold prices (Bottom left), and Crude Oil prices (Bottom right)

Even if the Markov switching gives the ability to capture differently in the market movement in different regimes, the coefficients of those variables in each state still appear to be varied over time. Figure 3 presents the jump out of the horizontal line during the period of 1997-2002, 2009-2010 and 2012-2013. We found that this model can methodologically capture the three real economic situations, namely Asian and Russian financial crisis in 1997-2002, the Hamburger crisis in 2009-2010 and the European debt crisis in 2012.

The transition probability matrix of time varying with Markov switching regression is given in Table 3. The transition probability is the conditional probability based on the information available throughout the whole sample period at future date $t$. The result shows that on the one hand the probability of switching from regime 1 (downturn market) to regime 2 (upturn market) is 0.015 , while in regime 1 is remaining 0.990 . On the other hand, the probability of switching from regime 2 to regime 1 is 0.010 while in regime 2 is remaining 0.985 .

Tables 3 Transition Proobability matrix (Time-varying MS regression)

\begin{tabular}{lcc}
\hline & Regime 1 & Regime 2 \\
\hline Regime 1 & 0.990 & 0.015 \\
Regime 2 & 0.010 & 0.985 \\
\hline
\end{tabular}

\section{Conclusions}

The paper introduces the Markov switching regression with time-varying coefficients as the contribution in the purpose of discussing the factors (gold prices and crude oil prices) affecting stock price index. The study applied Bayesian estimator to estimate the model parameters while the Kalman filter is applied to predict the time-varying coefficient in each regime. We found that the 
proposed model is able to capture the real economic situation very well particularly the crucial moment in 1997-2002, 2009-2010 and 2012. It can illustrate the relationship of gold price and crude oil price to stock market index. Therefore, this paper considerably suggests the use of Markov switching regression with time-varying coefficients when the research studies are purposed to deal with the high fluctuation data because it can capture the movement of the coefficients over time appropriately.

\section{Acknowledgement}

We thank to Graduate School and Faculty of Economics, Chiang Mai University (CMU) for financial support as well as participants at the International Conference on Economics, Finance and Statistics (ICEFS 2017) for time attention. Finally, thanks to Yamaka, W. for the valuable comment to improve this paper.

\section{References}

[1] I. Akgül, M. Bildirici and S. Özdemir. Evaluating the Nonlinear Linkage between Gold Prices and Stock Market Index Using Markov-Switching Bayesian VAR Models. Procedia - Social and Behavioral Sciences Vol. 210 (2015), p. 408-415

[2] S. Basher, A.A. Haug and P. Sadorsky. Oil prices, exchange rates and emerging stock markets. Energy Economics Vol. 34 (2012), p. 227-240

[3] J.Y. Philip, and R.J. Robert. Bond Market Volatility VS. Stock Market Volatility: The Swiss Experience, Swiss Society for Financial Market Research, (2004), p. 8-23

[4] C.J. Kim and C.R. Nelson. State-space models with regime switching: classical and Gibbs-sampling approaches with application, Cambridge, (1999)

[5] C.J. Kim. Dynamic Linear Models with Markov-switching, Journal of Econometrics (1994), p. 144-165

[6] A.J. Filardo. Choosing information Variables for transition probabilities in a time-varying transition probability Markov Switching model, Federal Reserve Bank of Kansas City, Vol. 98-09 (1998),

[7] P. Maneejuk, P. Pastpipatkul and S. Sriboonchitta. Analyzing the effect of Time-Varying Factors for Thai Rice Export, Thai Journal of Mathematics, (2016), p. 201-213

[8] K.H. Kang. Estimation of state space models with endogenous Markov regime switching parameters, The Econometrics Journal 17 (2014), p. 56-82

[9] M.West and J. Harrison: Baysian Forecasting and Dynamic Models, Springer Varlag, (1997)

[10] J.D. Hamilton. A New Approach to the Economic Analysis of Nonstationary Time Series and the Business Cycle, Econometrica 57 (1989), p. 357-384

[11]J.D. Hamiton. State-space models, Handbook of Econometrics 4 (1994), p. 3039-3080

[12] D. Wang and S.K. Ghosh. Bayesian analysis of random coefficient autoregessive models, Model Assisted Statistics and Applications 3 (2002), p. 281-295

[13] R.Jammazi. Oil shock transmission to stock market returns: Wavelet-multivariate Markov switching GARCH approach, Energy, Vol. 37 (2012), p. 430-454

[14]B. Miguel and K. Gary. Model Switching and Model Averaging in Time-Varying Parameter Regression Models, Scottish Institute for Research in Economics, (2013) 